\title{
Non-Analytic Tableaux for Chellas's Conditional Logic CK and Lewis's Logic of Counterfactuals VC
}

\author{
Richard Zach*
}

\begin{abstract}
Priest has provided a simple tableau calculus for Chellas's conditional logic $\mathbf{C k}$. We provide rules which, when added to Priest's system, result in tableau calculi for Chellas's CK and Lewis's VC. Completeness of these tableaux, however, relies on the cut rule.
\end{abstract}

\section{Introduction}

Chellas [1975] presented a conditional logic CK that is sound and complete for a relational semantics. The system uses an intensional conditional $\Rightarrow$ which plays the same role as the counterfactual conditional $\square \rightarrow$ of Lewis [1973]. Chellas realized that the conditional $\varphi \square \leftrightarrow \psi$ can be seen as a necessity operator in which the accessibility relation is indexed by the proposition expressed by the antecedent. He suggested the notation $[\varphi] \psi$. Lewis's dual might conditional $\varphi \diamond \leftrightarrow \psi$ would then correspond to an indexed possibility operator $\langle\varphi\rangle \psi$. Segerberg [1989] extended Chellas's semantics and showed that Lewis's logic of counterfactuals VC is an axiomatic extension of $\mathbf{C K}$ complete relative to the class of "Segerberg models" that satisfy certain restrictions.

In note 14 of his paper, Chellas [1975] suggests an alternative semantics in which the accessibility relation is not indexed by propositions but by formulas themselves. While in $\mathbf{C K}$ and its extensions $[\varphi] \theta$ is equivalent to $[\psi] \theta$

*University of Calgary, Department of Philosophy, 2500 University Dr NW, Calgary AB T2N 1N4, Canada, rzachucalgary.ca

Australasian Journal of Logic (15:3) 2018, Article no. 1 
whenever $\varphi$ and $\psi$ are equivalent (always true at the same worlds), this is not so when the accessibility relation is indexed by the formulas $\varphi$ and $\psi$. The result is a logic $\mathbf{C k}$ with a simpler semantics. It is characterized by the rules and axioms of $\mathbf{C K}$ without the rule RCEA. Priest [2008] discussed Chellas's Ck in more depth and provided a sound and cut-free complete tableau system for it.

We give additional branch extension rules for Priest's system which result in sound and complete tableau systems for $\mathbf{C K}$ and VC. These systems are, however, non-analytic in that the cut rule is included. The completeness proof proceeds by showing that tableau provability is closed under the rules and axioms of VC as given by Chellas and Segerberg.

These are not the first tableau systems for Lewis's VC. Gent [1992], based on work of de Swart [1983], has given a tableau system for VC. More recently, Girlando et al. [2016] and Negri and Sbardolini [2016] have offered cut-free complete sequent calculi for VC and others. These approaches are all based on Lewis's semantics based on relative proximity of worlds and incorporate the ordering relation between worlds into the syntax. The present approach is perhaps more perspicuous and holds promise for other logics based on the Chellas-Segerberg approach, such as those studied by Unterhuber [2013] and Unterhuber and Schurz [2014]. A related approach using labelled formulas was taken by Poggiolesi [2016] for an extension of CK, based on Nute's semantics. A major drawback of our proposal is of course the presence of the cut rule. Semantic proofs of cut-free completeness face significant challenges, which we discuss.

\section{Syntax and Semantics}

The syntax of Chellas's CK is that of propositional logic with the addition of an indexed necessity operator $[\varphi]$ :

Definition 1. Formulas are defined inductively:

1. Every propositional variable $p$ is a formula.

2. $\perp$ is a formula.

3. If $\varphi$ is a formula, so is $\neg \varphi$.

Australasian Journal of Logic (15:3) 2018 Article no. 1 
4. If $\varphi$ and $\psi$ are formulas, so are

$$
(\varphi \supset \psi),(\varphi \wedge \psi),(\varphi \vee \psi),[\varphi] \psi
$$

We can define $\top$ as $\neg \perp,\langle\varphi\rangle \psi$ as $\neg[\varphi] \neg \psi$, and $\varphi \equiv \psi$ as $(\varphi \supset \psi) \wedge(\psi \supset \varphi)$. We can also define $\square \varphi$ as $[\neg \varphi] \perp$ and $\diamond \varphi$ as $\langle\varphi\rangle \top$ (or, $\neg[\varphi] \perp$ ). If Lewis's notation is preferred, then $\operatorname{read} \varphi \square \mapsto \psi$ as $[\varphi] \psi$ and $\varphi \diamond \mapsto \psi$ as $\langle\varphi\rangle \psi$, i.e., $\neg[\varphi] \neg \psi$. Segerberg preferred the notations $\varphi \sqsupset \psi$ and $\varphi>\psi$ to Chellas's $[\varphi] \psi$ and $\langle\varphi\rangle \psi$.

Chellas also provided a relational semantics for which his logic $\mathbf{C K}$ is sound and complete. However, that semantics does not cover Lewis's VC. Segerberg provided the required generalization: a semantics for which the axiomatic system for $\mathbf{V C}$ is sound and complete.

Definition 2. A Segerberg model $M=\langle U, P, R, V\rangle$ consists of a set of worlds $U \neq \emptyset$, a set of propositions $P \subseteq \wp(U)$, a propositionally indexed accessibility relation $R: P \rightarrow \wp(U \times U)$, and a variable assignment $V: \operatorname{Var} \rightarrow$ $P$.

We write $R_{S}$ for $R(S)$ and $R_{S}[x]$ for $\left\{y \mid x R_{S} y\right\}$.

The set of propositions must contain $\emptyset$, be closed under intersection, complement, and necessitation, i.e.,

1. $\emptyset \in P, U \in P$.

2. If $S \in P$, then $U \backslash S \in P$.

3. If $S, T \in P$, then $S \cap T \in P$ and $S \cup T \in P$.

4. If $S, T \in P$, then $\left\{x \in U \mid R_{S}[x] \subseteq T\right\} \in P$.

Definition 3. Truth of a formula $\varphi$ at a world $x$ in $M, M, x \vDash \varphi$, is defined by:

1. $M, x \not \models \perp$ always.

2. $M, x \vDash p$ iff $x \in V(p)$.

3. $M, x \vDash \varphi \wedge \psi$ iff $M, x \vDash \varphi$ and $M, x \vDash \psi$.

4. $M, x \vDash \varphi \vee \psi$ iff $M, x \vDash \varphi$ or $M, x \vDash \psi$.

5. $M, x \vDash \varphi \supset \psi$ iff $M, x \not \vDash \varphi$ or $M, x \vDash \psi$.

Australasian Journal of Logic (15:3) 2018 Article no. 1 
6. $M, x \vDash[\varphi] \psi$ iff for all $y$ such that $x R_{\varphi} y, M, y \vDash \psi$.

7. $M, x \vDash\langle\varphi\rangle \psi$ iff for some $y$ such that $x R_{\varphi} y, M, y \vDash \psi$.

We write $\llbracket \varphi \rrbracket$ for $\{x \mid M, x \vDash \varphi\}$ and $R_{\varphi}$ for $R_{\llbracket \varphi \rrbracket}$.

We say that $M$ satisfies $\varphi$ if $M, x \vDash \varphi$ for some $x \in U ; M$ satisfies $\Gamma$ if for some $x \in U, M, x \vDash \varphi$ for all $\varphi \in \Gamma ; \Gamma$ is satisfiable if some $M$ satisfies $\Gamma$.

Definition 4. We say that $\Gamma \boldsymbol{C K}$-entails $\varphi, \Gamma \vDash_{\mathbf{C K}} \varphi$, iff for every Segerberg model $M$ and world $x$ such that $M, x \vDash \psi$ for every $\psi \in \Gamma, M, x \vDash \varphi$.

\section{Tableaux for $\mathrm{Ck}$ and $\mathrm{CK}$}

Definition 5. Prefixed formulas are expressions of the form $i: \varphi$ or $i \varphi j$.

Intuitively, $i: \varphi$ means " $\varphi$ is true at $i$ " and $i \varphi j$ means " $j$ is $\varphi$-accessible from $i . "$

Definition 6. Let $\Gamma$ be a set of prefixed formulas. A tableau is a downwardbranching tree labelled by prefixed formulas such that every prefixed formula in the tree is either in $\Gamma$ or is a conclusion of a branch extension rule. If a formula is one conclusion of a branching rule, its siblings in the tree must be labelled with the other conclusions.

A branch of a tableau is closed if it contains both $i: \varphi$ and $i: \neg \varphi$ for some $i$ and $\varphi$, or it contains $i: \perp$.

We write $\Gamma \vdash \varphi$ if $\{1: \psi \mid \psi \in \Gamma\} \cup\{1: \neg \varphi\}$ has a closed tableau.

We say a tableau is a $\mathbf{C k}$-tableau if it only uses the rules of Table 1, and a Vc-tableau if it also used the rules of Table 3. A CK- or VC-tableau is a Ck- or Vc-tableau that in addition uses the rules

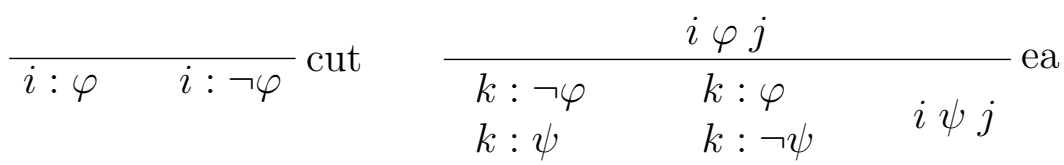

In the cut rule, the index $i$ must already occur on the branch. In the ea rule, the index $k$ must be new to the branch.

Both cut and ea are non-analytic in the sense that the conclusion formulas are not subformulas of the premises. This is an essential property of cut. The

Australasian Journal of Logic (15:3) 2018 Article no. 1 


$$
\begin{aligned}
& \begin{array}{ll}
i: \varphi \wedge \psi & \wedge \\
i: \varphi
\end{array} \quad \frac{i: \neg(\varphi \wedge \psi)}{i: \neg \varphi \quad i: \neg \psi} \neg \wedge \\
& \frac{i: \varphi \vee \psi}{i: \varphi \quad i: \psi} \vee \quad \frac{i: \neg(\varphi \vee \psi)}{i: \neg \varphi} \neg \vee \\
& \frac{i: \varphi \supset \psi}{i: \neg \varphi \quad i: \psi} \supset \quad \frac{i: \neg(\varphi \supset \psi)}{i: \varphi} \neg \supset \\
& \frac{i: \neg \neg \varphi}{i: \varphi} \neg \\
& i:[\varphi] \psi \\
& \frac{i \varphi j}{j: \psi} \square \quad \frac{i: \neg[\varphi] \psi}{j: \neg \psi} \neg \square \\
& \begin{array}{lc}
i:\langle\varphi\rangle \psi & i: \neg\langle\varphi\rangle \psi \\
j: \psi & \frac{i \varphi j}{j: \neg \psi} \neg \diamond
\end{array}
\end{aligned}
$$

Table 1: Branch extension rules for Priest's tableau system for Chellas's Ck

Australasian Journal of Logic (15:3) 2018 Article no. 1 
rule ea could, however, be replaced by the following analytic rule:

\begin{tabular}{|c|c|c|}
\hline \multicolumn{3}{|c|}{$i:[\psi] \theta$} \\
\hline$k: \neg \varphi$ & $k: \varphi$ & $j: \theta$ \\
\hline$k: \psi$ & $k: \neg \psi$ & $J \cdot 0$ \\
\hline
\end{tabular}

This rule could also replace the $\square$ rule in tableau systems for $\mathbf{C K}$ and VC.

We write $\Gamma \vdash_{\mathbf{C k}} \varphi$, etc., to indicate that there is a closed $\mathbf{C k}$-tableau that shows $\Gamma \vdash \varphi$.

Proposition 7. There are $\boldsymbol{C k}$-proofs of the following:

$$
\begin{aligned}
{[\varphi](\psi \wedge \theta) } & \vdash[\varphi] \psi \wedge[\varphi] \theta \\
{[\varphi] \psi \wedge[\varphi] \theta } & \vdash[\varphi](\psi \wedge \theta) \\
& \vdash[\varphi] \top
\end{aligned}
$$

Proof. See Appendix.

CM and CC together are Segerberg's M1, and CN is Segerberg's M2.

Proposition 8. If $\psi \vdash_{C k} \theta$, then $[\varphi] \psi \vdash_{C k}[\varphi] \theta$

Proof. Since $\psi \vdash_{\mathbf{C k}} \theta$, there is a closed $\mathbf{C k}$-tableau with assumptions 1 : $\psi, 1: \neg \theta$. If we raise every index in it by 1 it remains a closed tableau. Now consider:

$\begin{array}{lcl}\text { 1. } & 1:[\varphi] \psi & \text { Ass } \\ \text { 2. } & 1: \neg[\varphi] \theta & \text { Ass } \\ \text { 3. } & 1 \varphi 2 & 2 \neg \square \\ \text { 4. } & 2: \neg \theta & 2 \neg \square \\ \text { 5. } & 2: \psi & 1 \square \\ 6 . & \vdots & \\ & \otimes & \end{array}$

where the part indicated by $\vdots$ is the above closed tableau for $\psi \vdash \theta$ with indices raised by 1 but without its assumptions (which however appear on lines 4 and 5). Since that tableau closes, the resulting tableau closes.

Australasian Journal of Logic (15:3) 2018 Article no. 1 
This establishes the derivability of Chellas's rule RCEC, aka Segerberg's EC:

$$
\frac{\psi \equiv \theta}{[\varphi] \psi \equiv[\varphi] \theta} \text { RCEC }
$$

Proposition 9. If $\varphi \vdash_{\boldsymbol{C K}} \psi$ and $\psi \vdash_{\boldsymbol{C} \boldsymbol{K}} \varphi$, then $[\varphi] \theta \vdash_{\boldsymbol{C K}}[\psi] \theta$.

Proof. Consider the tableau

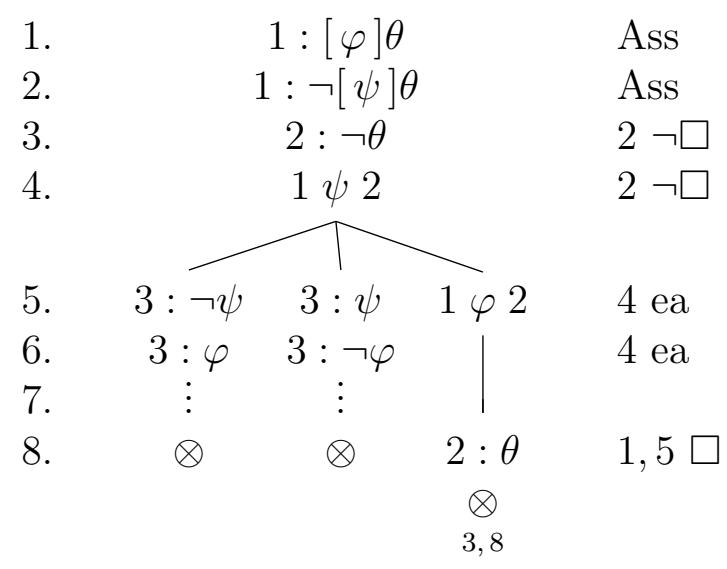

The parts indicated by $\vdots$ are the closed tableaux for $\varphi \vdash \psi$ and $\psi \vdash \varphi$, respectively, with all indices raised by 2 and the assumption removed.

This establishes the derivability of Chellas's rule RCEA, aka Segerberg's EA:

$$
\frac{\varphi \equiv \psi}{[\varphi] \theta \equiv[\psi] \theta} \text { RCEA }
$$

Proposition 10. In the presence of cut, if $\Gamma \vdash \varphi$ and $\varphi \vdash \psi$, then $\Gamma \vdash \psi$.

Proof. If $\Gamma=\left\{\theta_{1}, \ldots, \theta_{n}\right\}, \Gamma \vdash \varphi$ means there is a closed tableau for

$$
1: \theta_{1}, \ldots, 1: \theta_{n}, 1: \neg \varphi \text {. }
$$

Using cut, we can construct a closed tableau for

$$
1: \theta_{1}, \ldots, 1: \theta_{n}, 1: \neg \psi
$$

as follows:

Australasian Journal of Logic (15:3) 2018 Article no. 1 


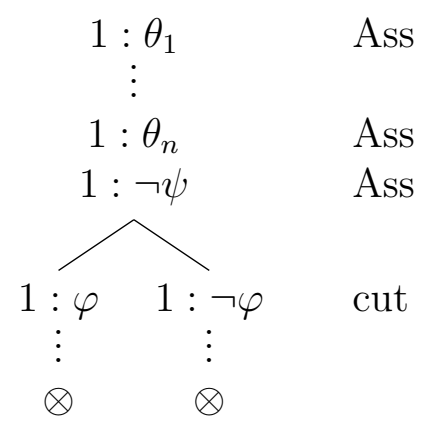

The sub-tableau on the left is the closed tableau for $\varphi \vdash \psi$, since that branch contains both $1: \varphi$ and $1: \neg \psi$. The one on the right is the closed tableau for $\Gamma \vdash \varphi$, since that branch contains all of $1: \theta_{1}, \ldots, 1: \theta_{n}, 1: \neg \varphi$.

Theorem 11. 1. If there is a derivation using RCEC and modus ponens using tautologies and axioms $C M, C C, C N$ of $\varphi$ from $\Gamma$, then there is a $\boldsymbol{C k}$-tableau with cut that shows $\Gamma \vdash_{C k+\text { cut }} \varphi$.

2. If there is a derivation using $R C E A, R C E C$, and modus ponens using tautologies and axioms $C M, C C, C N$ of $\varphi$ from $\Gamma$, then there is a $\boldsymbol{C K}$ tableau that shows $\Gamma \vdash_{C K} \varphi$.

Proof. 1. Propositional tautologies have closed tableaux since the rules for the ordinary propositional connectives are complete. The axioms CM, CC, CN have closed tableaux by Proposition 7. Closure of $\vdash_{\mathbf{C k}}$ under modus ponens is established by Proposition 10 (this requires cut). Closure under RCEC is established by Proposition 8.

2. Follows from (1) and Proposition 9.

Corollary 12. If $\Gamma \vDash_{C K} \varphi$ then $\Gamma \vdash_{C K} \varphi$.

Proof. Segerberg [1989] showed strong completeness of the system RCEA, RCEC, CM, CC, CN of CK for Segerberg models. The result follows by Theorem 11(2).

We defer proofs of soundness to Corollary 15. 


\begin{tabular}{l|l|l} 
& Axiom & Condition \\
\hline \hline S1 & {$[\varphi] \varphi$} & $R_{S}[x] \subseteq S$ \\
S2 & $\langle\varphi\rangle \psi \supset\langle\psi\rangle \top$ & $R_{S}[x] \cap T \neq \emptyset \Rightarrow R_{T}[x] \neq \emptyset$ \\
S3 & $\varphi \supset[\top] \varphi$ & $R_{U}[x] \subseteq\{x\}$ \\
S4 & $\varphi \supset\langle T\rangle \varphi$ & $x \in R_{U}[x]$ \\
S5 & {$[\varphi \wedge \psi] \theta \supset[\varphi](\psi \supset \theta)$} & $R_{S}[x] \cap T \subseteq R_{S \cap T}[x]$ \\
S6 & $\langle\varphi\rangle \psi \supset([\varphi](\psi \supset \theta) \supset$ & $R_{S}[x] \cap T \neq \emptyset \Rightarrow$ \\
& {$[\varphi \wedge \psi] \theta$} & $R_{S \cap T}[x] \subseteq R_{S}[x] \cap T$ \\
\hline
\end{tabular}

Table 2: Segerberg's axioms for VC and corresponding conditions

\section{The systems Vc and VC}

Segerberg [1989] has given an axiomatization of Lewis's logic VC of counterfactuals in the framework set out by Chellas, and proven completeness for Segerberg models that satisfy a number of conditions. The axioms that have to be added to $\mathbf{C K}$, and the corresponding conditions on the models, are given in Table 2. The corresponding tableau system is the tableau system for CK plus the additional rules given in Table 3 .

We call a Segerberg model which satisfies the conditions of Table 2 a $\boldsymbol{V} \boldsymbol{C}$-model.

We show that our tableau system for $\mathbf{V C}$ is sound and complete for VCmodels. For soundness, we have to extend the definition of satisfaction to prefixed formulas.

Definition 13. Suppose $M$ is a Segerberg model, $\Gamma$ is a set of prefixed formulas, and $f: I \rightarrow U$ where $I$ is the set of indices occurring in $\Gamma$. We say $M, f$ satisfies $\Gamma$ iff

1. If $i: \varphi \in \Gamma$, then $M, f(i) \vDash \varphi$.

2. If $i \varphi j \in \Gamma$, then $f(i) R_{\varphi} f(j)$.

Theorem 14. Tableaux for $\boldsymbol{V} \boldsymbol{C}$ are sound for $\boldsymbol{V} \boldsymbol{C}$-models, i.e., any set of formulas with a closed tableau is not satisfiable.

Proof. We show that if a satisfiable branch is extended by an application of a rule, at least one resulting branch is satisfiable. Thus, every tableau starting

Australasian Journal of Logic (15:3) 2018 Article no. 1 


$$
\begin{array}{lc}
\frac{i \varphi j}{j: \varphi} \mathrm{R} 1 & i \top i \\
\mathrm{R} 4 \\
j: \psi & j: \psi \\
\frac{i \varphi j}{i \psi k} \mathrm{R} 2 & \frac{i \varphi j}{i \varphi \wedge \psi j} \mathrm{R} 5 \\
i: \varphi & j: \psi \\
j: \neg \varphi & i \varphi j \\
\frac{i \top j}{j: \varphi} \mathrm{R} 3 & \frac{i \varphi \wedge \psi k}{k: \psi} \mathrm{R} 6 \\
& i \varphi k
\end{array}
$$

In $\mathrm{R} 2, k$ must be new to the branch. In $\mathrm{R} 4, i$ must occur on the branch.

Table 3: Branch extension rules for VC

from a satisfiable set of assumptions $\Delta$ contains at least one satisfiable branch and thus cannot be closed.

If $\Delta$ is satisfiable, then for some $M$ and $x \in U, M, x \vDash \varphi$ for all $\varphi \in \Delta$. Let $f:\{1\} \rightarrow U$ be given by $f(1)=x$. Then $M, f$ satisfies the assumptions $1: \theta_{1}, \ldots, 1: \theta_{n}$ of the tableau where $\Delta=\left\{\theta_{1}, \ldots, \theta_{n}\right\}$.

Now let $\Gamma$ be the set of prefixed formulas on a branch satisfied by $M, f$. The cases for the rules given in Table 1 are routine; we carry out the cases for $[\varphi] \psi$ and $\neg[\varphi] \psi$ :

We reduce $i:[\varphi] \psi \in \Gamma$. The resulting branch is $\Gamma \cup\{j: \psi\}$ for some $j$ such that $i \varphi j \in \Gamma$. Since $M, f$ satisfies $\Gamma, M, f(i) \vDash[\varphi] \psi$ and $f(i) R_{\varphi} f(j)$. Hence, $M, f(j) \vDash \psi$, i.e., $M, f$ satisfies $j: \psi$.

We reduce $i: \neg[\varphi] \psi \in \Gamma$. The resulting branch is $\Gamma \cup\{i \varphi j\} \cup\{j: \neg \psi\}$ for some $j$ not occurring in $\Gamma$. Since $M, f$ satisfies $\Gamma, M, f(i) \not \models[\varphi] \psi$, there is some $y \in U$ with $f(i) R_{\varphi} y$ such that $M, y \not \models \psi$. Extend $f$ to $f^{\prime}$ by $f(j)=y$. Then $M, f^{\prime}$ also satisfies $\Gamma . M, f^{\prime}(j) \not \models \psi$, i.e., $M, f$ satisfies $j: \neg \psi \cdot f^{\prime}(i) R_{\varphi} f^{\prime}(j)$ by definition of $f^{\prime}$, so $M, f^{\prime}$ satisfies $i \varphi j$.

The cut rule is sound: Suppose a branch $\Gamma$ is extended by applying cut. We obtain two new branches, $\Gamma \cup\{i: \varphi\}$ and $\Gamma \cup\{i: \neg \varphi\}$, where $i$ already occurs in $\Gamma$. Since $M, f$ satisfies $\Gamma, f(i)$ is defined. Either $M, f(i) \vDash \varphi$ or 
$M, f(i) \not \models \varphi$. In the first case, $M, f$ satisfies $\Gamma \cup\{i: \varphi\}$; in the second, it satisfies $\Gamma \cup\{i: \neg \varphi\}$.

Rule ea is sound. Suppose $\Gamma$ contains $i \varphi j$. Since $M, f$ satisfies $\Gamma$, $f(i) R_{\varphi} f(j)$. If $\llbracket \varphi \rrbracket=\llbracket \psi \rrbracket$, then $R_{\psi}=R_{\varphi}$ and we have $f(i) R_{\psi} f(j)$, i.e., $M, f$ satisfies $i \psi j$. Otherwise, there is some $y \in U$ where $M, y \not \models \varphi$ but $M, y \vDash \psi$ or $M, y \vDash \varphi$ but $M, y \not \models \psi$. Extend $f$ to $f^{\prime}$ with $f^{\prime}(k)=y$ (since $k$ does not occur in $\Gamma$, this is possible). Then either $M, f^{\prime}$ satisfies $\Gamma \cup\{k: \neg \varphi, k: \psi\}$ or it satisfies $\Gamma \cup\{k: \varphi, k: \neg \psi\}$.

Now consider the rules of Table 3 and assume that $M$ satisfies the respective condition of Table 2 .

R1. $\Gamma$ contains $i \varphi j$ and the resulting branch contains $j: \varphi$. By condition (1), $R_{\varphi}[f(i)] \subseteq \llbracket \varphi \rrbracket$. Since $M, f$ satisfies $i \varphi j$ we have $f(i) R_{\varphi} f(j)$, i.e., $f(j) \in R_{\varphi}[f(i)]$. Thus, $M, f(j) \vDash \varphi$.

R2. $\Gamma$ contains $i \varphi j$ and $j: \psi$ and the extended branch contains $i \psi k$, where $k$ does not occur in $\Gamma$. Since $M, f$ satisfies $i \varphi j$ we have $f(i) R_{\varphi}$ $f(j)$, i.e., $f(j) \in R_{\varphi}[f(i)]$. Since $M, f$ satisfies $j: \psi$ we have $f(j) \in \llbracket \psi \rrbracket$, so $f(j) \in R_{\varphi}[f(i)] \cap \llbracket \psi \rrbracket$. By condition $(2), R_{\psi}[f(i)] \neq \emptyset$, i.e., there is some $y \in U$ such that $f(i) R_{\psi} y$. Extend $f$ to $f^{\prime}$ with $f^{\prime}(k)=y . M, f^{\prime}$ satisfies $i \psi k$.

R3. $\Gamma$ contains $i \top j ; i: \varphi$; and $j: \neg \varphi$. The resulting branch also contains $j: \varphi$ and is thus closed. So in this case we have to show that $\Gamma$ is not satisfiable. If $M, f$ satisfies $i \top j$ we have $f(i) R_{\top} f(j)$, i.e., $f(j) \in R_{\top}[f(i)]$. By condition $(3), f(i)=f(j)$. But this is impossible since $M, f(i) \vDash \varphi$ and $M, f(j) \vDash \neg \varphi$.

R4. In this case, $\Gamma$ is extended by adding $i \top i$. By condition (4), $f(i) \in$ $R_{U}[f(i)]$, and since $\llbracket \top \rrbracket=U$ we have $f(i) R_{\top} f(i)$.

R5. $\Gamma$ contains $i \varphi j$ and $j: \psi$ and is extended by $i \varphi \wedge \psi j$. Since $f(j) \in$ $R_{\varphi}[f(i)]$ and $f(j) \in \llbracket \psi \rrbracket$, by $(5)$ we get $f(j) \in R_{\varphi \wedge \psi}[f(i)]$, i.e., $f(i) R_{\varphi \wedge \psi}$ $f(j)$.

R6. $\Gamma$ contains $i \varphi j ; j: \psi$; and $i \varphi \wedge \psi k$, and is extended by $i \varphi k$ and $k: \psi$. Since $M, f$ satisfies $\Gamma$, we have $f(j) \in R_{\varphi}[f(i)]$ and $f(j) \in \llbracket \psi \rrbracket$. So $R_{\varphi}[f(i)] \cap \llbracket \psi \rrbracket \neq \emptyset$. By condition $(6), R_{\varphi \wedge \psi}[f(i)] \subseteq R_{\varphi}[f(i)] \cap \llbracket \psi \rrbracket$. In other words, for any $y$ such that $f(i) R_{\varphi \wedge \psi} y$ we have both $f(i) R_{\varphi} y$

Australasian Journal of Logic (15:3) 2018 Article no. 1 
and $y \in \llbracket \psi \rrbracket$. Since $M, f$ satisfies $i \varphi \wedge \psi k \in \Gamma$, we have that $f(i) R_{\varphi \wedge \psi}$ $f(k)$, i.e., $f(k)$ is such a $y$. Thus, $f(i) R_{\varphi} f(k)$ and $M, f(k) \vDash \psi$.

Corollary 15. Tableaux for $\boldsymbol{C k}$ and $\boldsymbol{C K}$ are sound for Segerberg models. Tableaux for $\boldsymbol{V} \boldsymbol{c}$ are sound for $\boldsymbol{V} \boldsymbol{C}$-models

Proof. The conditions for VC-models are only used in the verification of soundness of rules R1-6. Since VC is sound for VC-models, and Vc has fewer rules than $\mathbf{V C}, \mathbf{V c}$ is also sound for $\mathbf{V C}$-models.

Definition 16. We say that $\Gamma \boldsymbol{V} \boldsymbol{C}$-entails $\varphi, \Gamma \vDash_{\mathbf{V C}}$, iff for every VCmodel $M$ and world $x$ such that $M, x \vDash \psi$ for every $\psi \in \Gamma, M, x \vDash \varphi$.

Corollary 17. If $\Gamma \vdash_{V C} \varphi$ then $\Gamma \vDash_{V C} \varphi$.

Proposition 18. Tableaux for $\boldsymbol{V} \boldsymbol{C}$ are complete, i.e., if $\Gamma \vDash_{\boldsymbol{V} \boldsymbol{C}} \varphi$ then there is a closed $\boldsymbol{V} \boldsymbol{C}$-tableau for $\Gamma \vdash_{\boldsymbol{V} C} \varphi$.

Proof. Segerberg [1989] stated strong completeness of RCEC, RCEA, CM, CC, CN plus axioms S1-6 of Table 2. By Theorem 11, it suffices to show that axioms S1-6 have closed VC-tableaux. These can be found in the Appendix.

Finally, a remark about the system C2 of Stalnaker and Thomason [1970]. Also known as VCS, it is the logic characterized by Lewis as VC plus conditional excluded middle, $(\varphi \square \mapsto \psi) \vee(\varphi \square \mapsto \neg \psi)$, or, in Chellas's notation: $[\varphi] \psi \vee[\varphi] \neg \psi$. Segerberg frames for it are characterized by the condition that whenever $x R_{S} y$ and $x R_{S} z$, then $y=z$. A tableau rule for it would be

$$
\begin{aligned}
& i \varphi j \\
& i \varphi k \\
& j: \psi \\
& \frac{k: \psi}{\mathrm{cem}}
\end{aligned}
$$

The rule is clearly sound. Its addition results in a system complete for VCS, as it can prove conditional excluded middle:

Australasian Journal of Logic (15:3) 2018 Article no. 1 


$\begin{array}{lcl}\vdash[\varphi] \psi \vee[\varphi] \neg \psi & \\ \text { 1. } & 1: \neg([\varphi] \psi \vee[\varphi] \neg \psi) & \text { Ass } \\ \text { 2. } & 1: \neg[\varphi] \psi & 1 \neg \vee \\ \text { 3. } & 1: \neg[\varphi] \neg \psi & 1 \neg \vee \\ \text { 4. } & 2: \psi & 2 \neg \square \\ 5 . & 1 \varphi 2 & 2 \neg \square \\ 6 . & 3: \neg \psi & 3 \neg \square \\ 7 . & 1 \varphi 3 & 3 \neg \square \\ 8 . & 3: \psi & 5,7,4 \mathrm{cem} \\ & \otimes & \end{array}$

\section{Challenges for Proofs of Cut-Free Complete- ness}

We have shown that $\mathbf{C K}$ and $\mathbf{V C}$ are sound and complete with respect to Segerberg models and VC-models, respectively. The completeness theorem relies on the presence of the cut rule. What are the prospects of proving completeness without the cut rule? First, let us review the proof of cut-free completeness for Ck due to Priest [2008].

Definition 19. A Priest model $M=\langle U, R, V\rangle$ consists of a set of worlds $U \neq$ $\emptyset$, an accessibility relation $R:$ Frm $\rightarrow \wp(U \times U)$ indexed by formulas, and a variable assignment $V: \operatorname{Var} \rightarrow \wp U$.

We write $R_{\varphi}$ for $R(\varphi)$ and $R_{\varphi}[x]$ for $\left\{y \mid x R_{\varphi} y\right\}$.

Definition 20. Truth of a formula $\varphi$ at a world $x$ in $M, M, x \vDash \varphi$, is defined exactly as for Segerberg models.

Theorem 21 (Priest 2008, §5.9). Ck-tableaux are sound for Priest models.

Proof. As in Theorem 14.

Theorem 22 (Priest 2008, §5.9). Ck-tableaux are complete for Priest models, i.e., if $\Delta$ has no closed $\boldsymbol{C k}$-tableau, then it has a Priest model.

Proof. Call a branch complete if for every rule that can be applied on the branch has been applied. Since any finite branch contains only finitely many prefixed formulas, it can be extended by applying all finitely many rules that

Australasian Journal of Logic (15:3) 2018 Article no. 1 
are applicable. So if $\Delta$ has no closed tableau, there is a tableau with $\Delta$ as assumptions which contains at least one complete open branch $\Gamma$.

Let $U$ be the set of indices occurring on $\Gamma$. Let

$$
V(p)=\{i \mid i: p \in \Gamma\}
$$

Set $i R_{\varphi} j$ iff $i \varphi j \in \Gamma$.

We show that if $i: \theta \in \Gamma$ then $M, i \vDash \theta$ and if $i: \neg \theta \in \Gamma$ then $M, i \not \models \theta$ by induction on $\theta$.

1. $\theta$ is atomic: If $i: p \in \Gamma, M, i \vDash p$ by definition of $V$. If $i: \neg p \in \Gamma$, then since $\Gamma$ is open, $i: p \notin \Gamma$. Thus $i \notin V(p)$, and $M, i \not \models p$.

2. $\theta \equiv \neg \varphi$ : Suppose $i: \neg \varphi \in \Gamma$. If $\varphi$ is of the form $\neg \psi$, since $\Gamma$ is complete, $i: \psi \in \Gamma$. By induction hypothesis, $M, i \vDash \psi$ and so $M, i \vDash \neg \neg \psi$, i.e., $M, i \vDash \neg \varphi$. If $\varphi$ is not of the form $\neg \psi$, the case $i: \neg \varphi \in \Gamma$ will be treated in one of the cases below. If $i: \neg \neg \varphi \in \Gamma$, then again $i: \varphi \in \Gamma$ and we have $M, i \not \models \neg \varphi$.

3. $\theta \equiv \varphi \wedge \psi$ : Suppose $i: \varphi \wedge \psi \in \Gamma$. Since $\Gamma$ is closed, $i: \varphi \in \Gamma$ and $i: \psi \in \Gamma$. By induction hypothesis, $M, i \vDash \varphi$ and $M, i \vDash \psi$, so $M, i \vDash \varphi \wedge \psi$. If $i: \neg(\varphi \wedge \psi) \in \Gamma$, then since $\Gamma$ is closed, either $i: \neg \varphi \in \Gamma$ or $i: \neg \psi \in \Gamma$. Thus, either $M, i \not \models \varphi$ or $M, i \not \models \psi$ by IH, and $M, i \not \models \varphi \wedge \psi$.

4. The cases for $\theta \equiv \varphi \vee \psi$ and $\theta \equiv \varphi \supset \psi$ are handled similarly.

5. $\theta \equiv[\varphi] \psi:$ Suppose $i:[\varphi] \psi \in \Gamma$. For every $j$ such that $i R_{\varphi} j$, $i \varphi j \in \Gamma$ by definition of $R_{\varphi}$. So for every $j$ such that $i R_{\varphi} j, j$ occurs in $\Gamma$. Since $\Gamma$ is complete, the $\square$ rule must have been applied on $\Gamma$ with index $j$, i.e., $j: \psi \in \Gamma$. By induction hypothesis, $M, j \vDash \psi$. Hence, $M, j \vDash[\varphi] \psi$.

Now suppose $i: \neg[\varphi] \psi \in \Gamma$. Since $\Gamma$ is complete, $j: \neg \psi \in \Gamma$ and $i R_{\varphi} j \in \Gamma$ for some $j$. By IH, $M, j \not \models \psi$. By definition of $R_{\varphi}, i R_{\varphi} j$. So, $M, i \not \models[\varphi] \psi$.

Australasian Journal of Logic (15:3) 2018 Article no. 1 


\begin{tabular}{ll}
\hline \hline$(1)$ & $R_{\varphi}[x] \subseteq \llbracket \varphi \rrbracket$ \\
$(2)$ & $R_{\varphi}[x] \cap \llbracket \psi \rrbracket \neq \emptyset \Rightarrow R_{\psi}[x] \neq \emptyset$ \\
$(3)$ & $R_{\top}[x] \subseteq\{x\}$ \\
$(4)$ & $x \in R_{\varphi}[x]$ \\
$(5)$ & $R_{\varphi}[x] \cap \llbracket \psi \rrbracket \subseteq R_{\llbracket \varphi \wedge \psi \rrbracket}[x]$ \\
(6) & $R_{\varphi}[x] \cap \llbracket \psi \rrbracket \neq \emptyset \Rightarrow R_{\varphi \wedge \psi}[x] \subseteq R_{\varphi}[x] \cap \llbracket \psi \rrbracket$ \\
\hline
\end{tabular}

Table 4: Conditions on Vc-models

Extending Priest's proof of cut-free completeness to Vc would involve a definition of a class of Priest models for which Vc-tableaux are sound and cut-free complete. The obvious approach would be to reformulate Segerberg's conditions for Priest models. Let's call a Priest model that satisfies the conditions of Table 4 a $\boldsymbol{V} \boldsymbol{c}$-model.

We would now have to show that the model constructed from a tableau branch which is also closed under rules R1-6 satisfies the corresponding property in Table 4.

Condition $\mathrm{R} 1$ poses no problem: We have to show that $R_{\varphi}[i] \subseteq \llbracket \varphi \rrbracket$ for all $\varphi$. Suppose $i R_{\varphi} j$. By definition of $R, i \varphi j \in \Gamma$. Since $\Gamma$ is complete, rule R1 has been applied on it, so $j: \varphi \in \Gamma$. By the result above, $M, j \vDash \varphi$, i.e., $j \in \llbracket \varphi \rrbracket$.

The same approach does not work for $\mathrm{R} 2\left(R_{\varphi}[i] \cap \llbracket \psi \rrbracket \neq \emptyset \Rightarrow R_{\psi}[i] \neq \emptyset\right)$. For suppose $j \in R_{\varphi}[i] \cap \llbracket \psi \rrbracket$, i.e., $i R_{\varphi} j$ and $M, j \vDash \psi$. By definition of $R$, $i \varphi j \in \Gamma$. However, $M, j \vDash \psi$ does not guarantee that $j: \psi \in \Gamma$. In fact, if $\psi$ is not a subformula of $\Delta, j: \psi$ is guaranteed not to be in $\Gamma$.

For (3), suppose $i R_{\top} j$. Then $i \top j \in \Gamma$. Completeness under R3 only rules out $i \neq j$ if $i: \psi \in \Gamma$ for some $\psi$, which is not guaranteed. Etc.

To extend the proof of cut-free completeness to $\mathbf{V C}$ with respect to $\mathbf{V C}$ models, we face an even more difficult obstacle. For VC models have their accessibility relation indexed by propositions, not formulas. So the definition of a Segerberg model $M$ from an open complete branch $\Gamma$ would have to define $R_{S}$ on the basis of which $i \varphi j$ are in $\Gamma$. We could do this only if we already knew which propositions $\varphi$ expresses in $M$-then we could say that if $S=\llbracket \varphi \rrbracket, i R_{S} j$ iff $i \varphi j \in \Gamma$. But of course we can't do this, since $M$-which determines $\llbracket \varphi \rrbracket$ - is not yet defined! An additional obstacle is that rule ea does not guarantee that $i \varphi j$ iff $i \psi j$ even when, for all $k, k: \varphi \in \Gamma$ iff $k: \psi \in \Gamma$.

Australasian Journal of Logic (15:3) 2018 Article no. 1 


\section{Conclusion}

The above considerations show that using the approach pioneered by Chellas and Segerberg, and recently extended significantly by Unterhuber and Schurz, point to a way of constructing analytic rules for conditional logics (if not analytic tableaux calculi). The rules given are relatively straightforward translations of conditions on the indexed accessibility relation into tableaux rules. A condition expressible by a universal formula, such as S1 $\left(\forall i \forall j\left(i R_{\varphi} j \supset j \in \llbracket \varphi \rrbracket\right)\right)$, translates into extension rules without conditions. Those involving existential quantifiers, such as S2, i.e.,

$$
\forall i \forall j\left(\left(i R_{\varphi} j \wedge j \in \llbracket \psi \rrbracket\right) \supset \exists k i R_{\psi} k\right),
$$

involve the introduction of prefixes new to the branch. It is plausible that a fragment of first-order logic can be identified such that any condition expressible in that fragment can be translated into a sound tableau rule. Identity (and uniqueness), e.g., the condition for C2 $\left(\forall i \forall j \forall k\left(\left(i R_{\varphi} j \wedge i R_{\varphi} k\right) \supset j=\right.\right.$ $k)$ ) is a bit trickier: here the resulting rule does not force $j=k$ but only that all formulas evaluate the same at $j$ and $k$, i.e., $j$ and $k$ are indiscernible. So, other systems can be dealt with in a similar manner, as long as they are characterized by accessibility relations expressible in the right way. The lack of a proof of cut-free completeness is of course unsatisfying. Having candidate rules as well as a tentative analysis of why available methods of establishing cut-free completeness fail perhaps points in a direction of solving this open problem. If nothing else, we have highlighted that Chellas's and Segerberg's approach to a semantics for conditionals has not yet been sufficiently exploited in the search for analytic proof systems for a class of logics that includes systems as important as Lewis's VC.

Acknowledgements. I'd like to thank the reviewer for the $A J L$ for their comments.

\section{References}

Brian F. Chellas. Basic conditional logic. Journal of Philosophical Logic, 4 (2):133-153, 1975. doi: 10.1007/BF00693270.

Harrie C. M. de Swart. A Gentzen- or Beth-type system, a practical decision procedure and a constructive completeness proof for the counterfactual

Australasian Journal of Logic (15:3) 2018 Article no. 1 
logics VC and VCS. Journal of Symbolic Logic, 48(1):1-20, 1983. doi: $10.2307 / 2273315$.

Ian Philip Gent. A sequent- or tableau-style system for Lewis's counterfactual logic VC. Notre Dame Journal of Formal Logic, 33(3):369-382, 1992. doi: 10.1305/ndjll/1093634402.

Marianna Girlando, Björn Lellmann, Nicola Olivetti, and Gian Luca Pozzato. Standard sequent calculi for lewis' logics of counterfactuals. In Loizos Michael and Antonis C. Kakas, editors, Logics in Artificial Intelligence, JELIA 2016. Proceedings, volume 10021 of Lecture Notes in Computer Science, pages 272-287, 2016. doi: 10.1007/978-3-319-48758-8_18.

David K. Lewis. Counterfactuals. Blackwell, 1973.

Sara Negri and Giorgio Sbardolini. Proof analysis for Lewis counterfactuals. Review of Symbolic Logic, 9(1):44-75, $2016 . \quad$ doi: $10.1017 / \mathrm{S} 1755020315000295$.

Francesca Poggiolesi. Natural deduction calculi and sequent calculi for counterfactual logics. Studia Logica, 104(5):1003-1036, Oct 2016. doi: 10.1007/s11225-016-9662-3.

Graham Priest. An Introduction to Non-Classical Logic: From If to Is. Cambridge University Press, Cambridge, 2nd edition, 2008.

Krister Segerberg. Notes on conditional logic. Studia Logica, 48(2):157-168, 1989. doi: 10.1007/BF02770509.

Robert C. Stalnaker and Richmond H. Thomason. A semantic analysis of conditional logic. Theoria, 36(1):23-42, 1970. doi: 10.1111/j.17552567.1970.tb00408.x.

Matthias Unterhuber. Possible Worlds Semantics for Indicative and Counterfactual Conditionals? A Formal Philosophical Inquiry Into ChellasSegerberg Semantics. Ontos, 2013. URL https://philpapers.org/rec/ UNTPWS.

Matthias Unterhuber and Gerhard Schurz. Completeness and correspondence in Chellas-Segerberg semantics. Studia Logica, 102(4):891-911, 2014. doi: $10.1007 / \mathrm{s} 11225-013-9504-5$.

Australasian Journal of Logic (15:3) 2018 Article no. 1 


\section{Appendix}

\section{Tableaux for Ck-axioms}

1. Ck-tableau for CM:

$$
[\varphi](\psi \wedge \theta) \vdash_{\mathbf{C k}}[\varphi] \psi \wedge[\varphi] \theta
$$

1. $1:[\varphi](\psi \wedge \theta) \quad$ Ass

2. $1: \neg([\varphi] \psi \wedge[\varphi] \theta) \quad$ Ass

3. $1: \neg[\varphi] \psi \quad 1: \neg[\varphi] \theta \quad 2 \neg \wedge$

4. $2: \neg \psi \quad 2: \neg \theta \quad 3 \neg \square$

5. $1 \varphi 2 \quad 1 \varphi 2 \quad 3 \neg \square$

6. $2: \psi \wedge \theta \quad 2: \psi \wedge \theta \quad 1,5 \square$

7. $2: \psi \quad 2: \psi \quad 6 \wedge$

8. $2: \theta \quad 2: \theta \quad 6 \wedge$

$\otimes \quad \otimes$

$7,4 \quad 8,4$

2. Ck-tableau for CC:

$[\varphi] \psi \wedge[\varphi] \theta \vdash_{\mathbf{C k}}[\varphi](\psi \wedge \theta)$

1. $1:[\varphi] \psi \wedge[\varphi] \theta \quad$ Ass

2. $1: \neg[\varphi](\psi \wedge \theta) \quad$ Ass

3. $1:[\varphi] \psi \quad 1 \wedge$

4. $1:[\varphi] \theta \quad 1 \wedge$

5. $\quad 1 \varphi 2 \quad 2 \neg \square$

6. $2: \neg(\psi \wedge \theta) \quad 2 \neg \square$

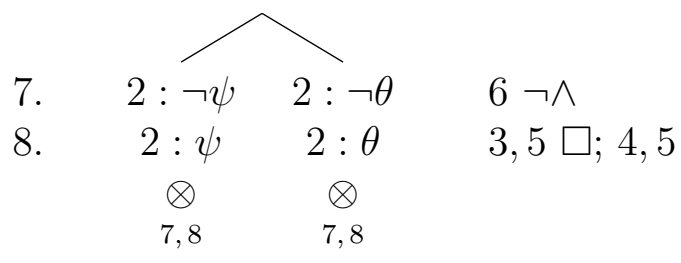

3. Ck-tableau for $\mathrm{CN}$ :

Australasian Journal of Logic (15:3) 2018 Article no. 1 
$\vdash[\varphi] \top$

1. $1: \neg[\varphi] \top \quad$ Ass

2. $1 \varphi 2 \quad 1 \neg \square$

3. $2: \neg \top \quad 1 \neg \square$

$\otimes$

3

\section{Vc-tableaux for axioms S1-6}

1. $\vdash[\varphi] \varphi$

1. $1: \neg[\varphi] \varphi \quad$ Ass

2. $1 \varphi 2 \quad 1 \neg \square$

3. $2: \neg \varphi \quad 1 \neg \square$

4. $2: \varphi \quad 2 \mathrm{R} 1$

$\otimes$

3,4

2. $\langle\varphi\rangle \psi \vdash\langle\psi\rangle \top$

1. $1:\langle\varphi\rangle \psi \quad$ Ass

2. $1: \neg\langle\psi\rangle \top \quad$ Ass

3. $1 \varphi 2 \quad 1 \diamond$

4. $2: \psi \quad 1 \diamond$

5. $1 \psi 2 \quad 3,4 \mathrm{R} 2$

6. $2: \neg \top \quad 2,5 \neg \diamond$

$\otimes$

6

3. $\varphi \vdash[\top] \varphi$

$\begin{array}{ccl}\text { 1. } & 1: \varphi & \text { Ass } \\ \text { 2. } & 1: \neg[\top] \varphi & \text { Ass } \\ \text { 3. } & 1 \top 2 & 2 \neg \square \\ \text { 4. } & 2: \neg \varphi & 2 \neg \square \\ \text { 5. } & 2: \varphi & 1,3,4 \mathrm{R} 3 \\ & \otimes & \\ & 4,5 & \end{array}$

Australasian Journal of Logic (15:3) 2018 Article no. 1 
4. $\varphi \vdash\langle\top\rangle \varphi$

$\begin{array}{lcl}\text { 1. } & 1: \varphi & \text { Ass } \\ \text { 2. } & 1: \neg\langle\top\rangle \varphi & \text { Ass } \\ \text { 3. } & 1 \top 1 & \text { R4 } \\ \text { 4. } & 1: \neg \varphi & 2,3 \neg \diamond \\ & \otimes & \\ & 1,4 & \end{array}$

5. $[(\varphi \wedge \psi)] \theta \vdash[\varphi](\psi \supset \theta)$

$\begin{array}{lcl}\text { 1. } & 1:[(\varphi \wedge \psi)] \theta & \text { Ass } \\ \text { 2. } & 1: \neg[\varphi](\psi \supset \theta) & \text { Ass } \\ \text { 3. } & 1 \varphi 2 & 2 \neg \square \\ \text { 4. } & 2: \neg(\psi \supset \theta) & 2 \neg \square \\ \text { 5. } & 2: \psi & 4 \neg \supset \\ \text { 6. } & 2: \neg \theta & 4 \neg \supset \\ \text { 7. } & 1 \varphi \wedge \psi 2 & 3,5 \mathrm{R} 5 \\ \text { 8. } & 2: \theta & 1,7 \square \\ & \otimes & \end{array}$

6. $\langle\varphi\rangle \psi,[\varphi](\psi \supset \theta) \vdash[\varphi \wedge \psi] \theta$

\begin{tabular}{lcl} 
1. & $1:\langle\varphi\rangle \psi$ & Ass \\
2. & $1:[\varphi](\psi \supset \theta)$ & Ass \\
3. & $1:[\varphi \wedge \psi] \theta$ & Ass \\
4. & $1 \varphi 2$ & $1 \diamond$ \\
5. & $2: \psi$ & $1 \diamond$ \\
6. & $1(\varphi \wedge \psi) 3$ & $3 \neg \square$ \\
7. & $3: \neg \theta$ & $3 \neg \square$ \\
8. & $1 \varphi 3$ & $4,5,6 \mathrm{R} 6$ \\
9. & $3: \psi$ & $4,5,6 \mathrm{R} 6$ \\
10. & $3: \psi \supset \theta$ & $2,8 \square$ \\
\multicolumn{4}{c}{} \\
11. & $3: \neg \psi \quad 3: \theta$ & $10 \supset$ \\
& $\otimes$ & $\otimes$
\end{tabular}

Australasian Journal of Logic (15:3) 2018 Article no. 1 